\title{
General
}

\section{Relapse of Psychosis Status Post Meningioma Resection}

\author{
Hamza Mohammad-Amin, MD ${ }^{1}$, Amber N. Edinoff, MD¹ ㅇ, Haseeb A. Akuly, DO', Prithvi Doppalapudi, MD¹, Rita \\ Horton, MD', Anthony Sin, MD', Donard Dwyer, PhD', City Mohammad Amin', Sana Shamim \\ 1 Department of Psychiatry and Behavioral Medicine, Louisiana State University Health Shreveport \\ Keywords: visual hallucinations, auditory hallucinations, meningiomas, psychosis \\ https://doi.org/10.52965/001c.27359
}

\section{Health Psychology Research}

Vol. 9, Issue 1, 2021

\begin{abstract}
A 53-year-old Caucasian female with a previous psychiatric history of bipolar I disorder and attention deficit hyperactivity disorder presented to the emergency department after endorsing 10-11 months of auditory and visual hallucinations, persecutory delusions, depression, anosmia, weakness of lower extremities, and headache. The patient described her auditory hallucinations as non-commanding voices talking to her about her family, her visual hallucinations as seeing "shadows and shapes," and her paranoid delusions as people coming after her. The patient had sustained a fall a week and a half earlier, requiring eight sutures to her posterior scalp. Her MRI of the brain showed a well-circumscribed $3.5 \times 4.7 \times 3.2 \mathrm{~cm}$ mass in the floor of the anterior cranial fossa. Computer tomography of the brain showed a $4.5 \mathrm{~cm}$ mass near the anterior interhemispheric fissure and edema in the right frontal cortex. Meningioma resection resulted in the cessation of hallucinations and delusions for one week. However, the patient was brought back to the emergency department because her auditory hallucinations and delusions returned. This case report demonstrates residual psychosis, even after frontal meningioma resection.
\end{abstract}

\section{INTRODUCTION}

Meningiomas are tumors that arise from the three membranes (meninges) surrounding the brain and the spinal cord. Although mostly benign (90\%), meningiomas comprised $33.8 \%$ of all primary brain and central nervous system tumors in the United States from 2002 to 2006. ${ }^{1}$ The annual incidence rate of meningiomas in the United States has been $5 / 100,000 .^{2}$ Meningiomas are slow-growing tumors with insidious onset. ${ }^{3}$ Many meningioma patients do not present with neuropsychiatric symptoms until after the tumor's size produces a mass effect on the surrounding brain structures.

The locations of meningiomas generally determine the neuropsychiatric symptomatology. For instance, meningiomas near the frontal lobe typically result in several neuropsychiatric symptoms such as anosmia, headache, blurry vision, and auditory hallucinations. This case report presents the question of whether or not a patient's psychiatric symptoms of hallucinations and delusions will re-appear even after resection of a meningioma large enough to cause a mass effect. This is a case study of an individual who had hallucinations and delusions concurrent with a meningioma and residual symptoms post-resection.

\section{CASE REPORT}

A 53-year-old Caucasian female with a previous psychiatric history of bipolar I disorder and attention deficit hyperactivity disorder presented to the ED for neuropsychiatric symptoms of auditory hallucinations (multiple, non-commanding, threatening, male and female voices of 10-11 months duration), visual hallucinations (seeing "shadows and shapes" of 10-11 months duration), persecutory delusions (believing people were coming after her and thought the neurosurgeon had placed a chip in her head), depression, headaches, weakness of lower extremities, and anosmia. The patient had a 20-year history of bipolar I disorder, for which she was treated with and stabilized with several mood stabilizers and antidepressants. Her hallucinations and delusions only presented 10-11 months before her presentation. Three months before her ED visit, she had also been prescribed risperidone by a psychiatrist, for her auditory and visual hallucinations, with minimal relief. In addi-

\footnotetext{
a Corresponding author:

Dr. Hamza Mohammad-Amin, MD

Louisiana State University Health Science Center Shreveport

Department of Psychiatry and Behavioral Medicine

1501 Kings Hwy Shreveport, LA 71103

Phone: (318) 675-8969
} 
tion, 10 days before presenting to the ED, the patient had eight sutures placed to her posterior scalp for a laceration from a head injury due to a fall. On the day of presentation to the ED, a CT scan of the head without contrast and MRI of the brain with and without contrast were obtained in the ED for further testing. The CT demonstrated a $4.5 \mathrm{~cm}$ mass near the anterior interhemispheric fissure and edema in the right frontal lobe, and the MRI demonstrated a well-circumscribed $3.5 \times 4.7 \times 3.2 \mathrm{~cm}$ mass in the floor of the anterior cranial fossa. The neurosurgeon's mass assessment was that it had been present for "at least several years" without the patient being aware. The patient was transported to another hospital for a higher level of care for intracranial neoplasm excision. Pathological examination by frozen specimen confirmed that this was a meningioma. The meningioma was successfully resected within several days after the patient's diagnosis. Eventually, the patient was discharged with risperidone as her antipsychotic. The week following the resection, there was a complete cessation of the patient's hallucinations and delusions. However, after the initial resolution of the psychotic symptoms, the patient presented to the ED again with auditory hallucinations and delusions despite being compliant with her risperidone. The patient heard voices saying derogatory statements about her body, her family, and making sexual threats against her. She also called the police to check on her home because she started having paranoid and persecutory delusions that people were in the crawl spaces under her house. In addition, she started believing that a microchip was placed in the back of her head that was reading her mind and controlling her thoughts.

Consequently, the patient was admitted to an inpatient psychiatric facility in the hospital and restarted on risperidone. After spending a week in an inpatient setting, her auditory hallucinations and delusions were controlled with risperidone. She was discharged home with a prescription for risperidone and a referral to an outpatient mental health clinic for follow-up.

\section{DISCUSSION}

Frontal meningiomas can cause and mimic psychosis due to their mass effect on the surrounding brain structures. This case report not only demonstrates that a frontal meningioma can elicit psychosis, but it may also leave a lasting constellation of psychiatric symptoms such as auditory hallucinations and delusions, even after resection. In this patient, post-resection MRI and CT scans of the head showed a small residual interhemispheric subarachnoid hemorrhage, small right frontal subdural hematoma, and a mild-to-moderate bifrontal pneumocephalus. A follow-up CT brain of the head a month later showed near resolution of the right frontal subdural hematoma and complete resolution of both the previously seen subarachnoid hemorrhage and the pneumocephalus. However, despite these findings, the patient continued to have auditory hallucinations and delusions. This continuation demonstrates the possibility that the previous meningioma's mass effect may have left lasting psychiatric symptoms. Later, the patient's hallucinations and delusions were ultimately controlled by her daily intake of risperidone.

In other case studies, frontal meningiomas have been reported to have left enduring psychiatric symptoms, even after resection. In a case published by Coetzer, ${ }^{4}$ a woman in her mid-60's with auditory hallucinations presented to the hospital for worsening neurological symptoms of headaches, lethargy, and left leg weakness. An MRI was obtained, which showed a frontal lobe meningioma, which was ultimately resected, and a repeat scan 9 months later showed no recurrence of the meningioma. Her auditory hallucinations, however, did not go into remission even after compliance with her psychotropics. Another case report by Keshavan et al. ${ }^{5}$ demonstrated that a 43 -year-old man presented to the hospital for neurological symptoms of headaches, nausea, vomiting, and generalized leg weakness and was later found to have a frontal lobe meningioma. After his tumor was removed, he started presenting with symptoms of delusions, depression, and auditory and visual hallucinations of derogatory content. Although most of his symptoms improved after being placed on psychotropics, his delusions and auditory hallucinations worsened, and he started hearing an orchestra in his head playing "monotonous march type of music." Both case studies demonstrate that frontal meningiomas can cause long-lasting psychiatric symptoms, even after removal.

Regarding this specific case, we continue to question if the residual hallucinations and delusions reflected a learned component to a longstanding psychiatric illness that was only temporarily affected by resectioning the tumor. Even though she was prescribed risperidone and it had been recently refilled before her presentation, we cannot be sure that she was compliant with the medication. Alternatively, perhaps the meningioma was suppressing more severe symptomatology inherent to the course of the patient's bipolar disorder; removing the meningioma revealed this basal dysfunction.

Meningiomas may be present without a patient's awareness due to their slow-growing nature. Most patients with developing frontal meningiomas may present with neurological symptoms of headaches, anosmia, extremity weakness, visual defects, and psychiatric symptoms such as auditory hallucinations, visual hallucinations, and delusions. It is imperative, therefore, to obtain a thorough psychiatric and medical history. It is also important to include CNS masses in the differential for these patients and consider screening for intracranial masses via CT or MRI if indicated. In addition, there may be a correlation between lasting psychiatric symptoms and patients with meningiomas even after resection of the meningiomas. Therefore, a physician should convey this information to their patients and encourage them to seek psychiatric help if their symptoms deteriorate.

Submitted: June 30, 2021 EST, Accepted: July 25, 2021 EST 


\section{REFERENCES}

1. Wiemels J, Wrensch M, Claus EB. Epidemiology and etiology of meningioma. Journal of neuro-oncology. 2010;99:314.

2. Apra C, Peyre M, Kalamarides M. Current treatment options for meningioma. Expert Review of Neurotherapeutics. 2018;18(3):241-249. doi:10.1080/1 4737175.2018 .1429920

3. Kauke M, Safi AF, Stavrinou P, Krischek B, Goldbrunner R, Timmer M. Does Meningioma Volume Correlate with Clinical Disease Manifestation Irrespective of Histopathologic Tumor Grade? Journal of Craniofacial Surgery. 2019;30(8):e799-e802. doi:1 $\underline{0.1097 / \text { scs } .0000000000005845}$
4. Coetzer R. Auditory Hallucinations Secondary to a Right Frontal Meningioma. Journal of Neuropsychiatry and Clinical Neurosciences. 2014;26(3):E15. doi:10.117 6/appi.neuropsych.13060142

5. Keshavan MS, Kahn EM, Brar JS. Musical hallucinations following removal of a right frontal meningioma. Journal of Neurology, Neurosurgery \& Psychiatry. 1988;51(9):1235-1236. doi:10.1136/jnnp.5 1.9.1235 